\title{
Dependence of Josephson Junction Critical Current on The Deposition Rate of $\mathrm{YBa}_{2} \mathrm{Cu}_{3} \mathrm{O}_{7-\delta}$ Thin Films
}

\author{
B. P. Algul, I. Avci, R. Akram, A. Bozbey*, M. Tepe** and D. Abukay \\ Izmir Institute of Technology, 35437-Urla, Izmir, Turkey \\ *Bilkent University, Ankara, Turkey \\ **Ege University, Izmir, Turkey
}

\begin{abstract}
We have reported the effect of $\mathrm{YBa}_{2} \mathrm{Cu}_{3} \mathrm{O}_{7-\delta}$ (YBCO) thin film deposition rate on the 24 and 30 degree STO bicrystal Josephson junctions critical currents by fabricating series of junctions with different deposition rates. Dependence of YBCO thin film structures on the deposition rate was investigated. We have observed that the critical currents of junctions are strongly affected by the thin film deposition rate.
\end{abstract}

Keywords: Josephson Junctions, Deposition Rate

PACS: 85.25.Cp

\section{INTRODUCTION}

In the high temperature superconducting electronic devices based on Josephson junctions, the device properties $[1,2]$ depending on the thin film structure and fabrication process [3] have been widely studied. In this study we have investigate the influence of film fabrication process on such devices.

\section{EXPERIMENTAL RESULTS}

Superconducting $\mathrm{YBa}_{2} \mathrm{Cu}_{3} \mathrm{O}_{7-\delta}$ thin films, having different deposition rates and $150 \mathrm{~nm}$ thickness, have been deposited using dc magnetron sputtering. The junctions were patterned as $3 \mu \mathrm{m}$-width line on the grain boundary using standard photolithography process as seen in Fig. 1.

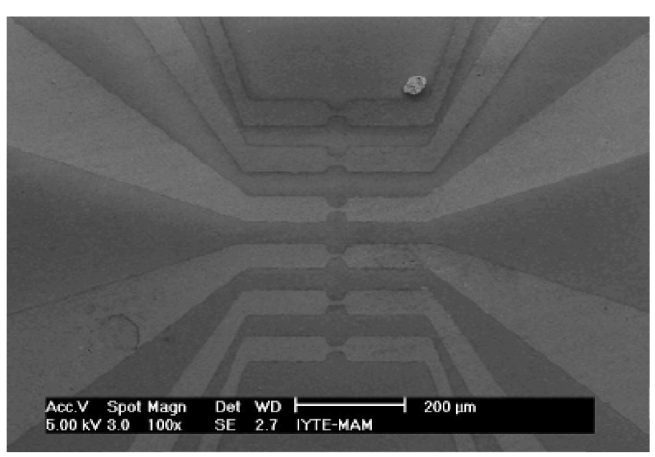

FIGURE 2. SEM Image of YBCO Josephson Junctions
In our study, we have observed that the critical currents, $I_{c}$, of junctions are changing by the $\mathrm{YBCO}$ thin film deposition rate as $I_{c}$ ranging from 10 to $500 \mu \mathrm{A}$ as indicated in Fig. 2 .

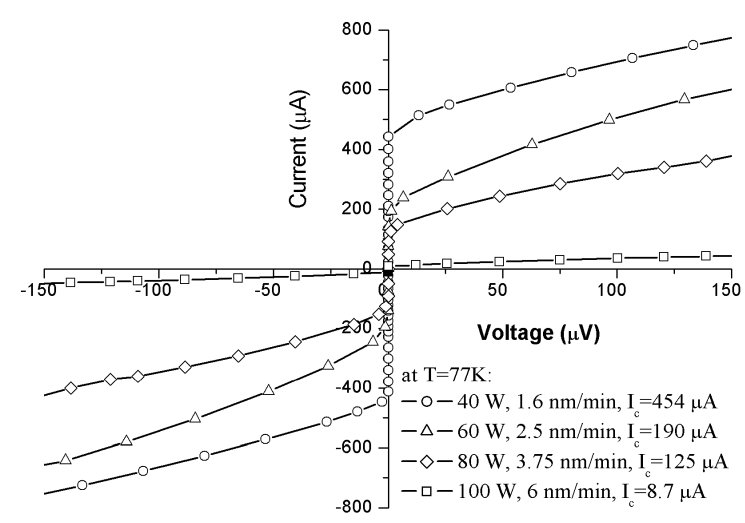

FIGURE 2. The Dependence of The Critical Currents on Deposition Rates of $3 \mu \mathrm{m}$-wide YBCO Josephson Junctions

\section{REFERENCES}

1. E. Sarnelli, G. Testa, Physica C 371 (2002) 10-18

2. M Esserst. P H Kest. W T Fu and H W Zandberaen Supercond. Sci. Technol. 6 (1993) 250-256.

3. Liu X.Z.; Li Y.R.; Tao B.W.; Luo A.; He S.M.M. P. Physica C, 371-2, (2002), 133-138(6) 